\title{
Ion sputtering of nanoclusters, fullerenes and carbon nanotubes
}

\author{
S. N. Kapustin ${ }^{1}$, M. K. Eseev ${ }^{1,2}$ \\ ${ }^{1}$ Northern Arctic Federal University named after M. V. Lomonosov, Arkhangelsk, Russia \\ ${ }^{2}$ Laverov Federal Center for Integrated Arctic Research RAS, Arkhangelsk, Russia \\ hare22@yandex.ru,m_eseev@mail.ru
}

PACS 79.20.Rf

DOI 10.17586/2220-8054-2018-9-1-129-131

\begin{abstract}
The research of nanoparticle ion sputtering is interesting both from the fundamental point of view - for researching the interior structure of nanoobjects. Additions to the simple model of ion sputtering which allow one to consider special properties of the ion sputtering of nanoparticles, fullerenes and carbon nanotubes are introduced in this project.
\end{abstract}

Keywords: carbon nanotube, fullerene, ion sputtering, clusters, nanoparticles.

Received: 20 June 2017

Revised: 16 October 2017

\section{Collisions of ions and nanoparticles}

In the work [1] a model of metal ion sputtering based on simple physical principles was consequently developed. This model allows one to evaluate mass, charge and kinetic spectra of polyatomic clusters with an atom number of $4<N<70$ when the deposited ion's ranges in energy from 1-100 keV. The model also describes the sputtering of nonmetallic elements, particularly, carbon and silicon.

The passage of an ion through a nanoparticle is generally studied using methods of molecular dynamics $[2,3]$. As an experimental work, this is very difficult because of the size of the target. The main characteristic of the ion sputtering process is the $Y$ coefficient of sputtering, equal to the number of atoms knocked out by one deposited ion. If the length of free run of the bombing ion in the target $(\lambda)$ is smaller than the cluster size $(D)$, the $\mathrm{Y}$ value for the cluster is considered bigger than that for macro bodies. The reason for this is the closure of the collisions cascade in the small volume of the cluster and the big surface area, from which an emission of secondary clusters is possible. This has been confirmed by the work [2]. The cases when $D<\lambda$ and $D \sim \lambda$ are studied in the work [3]. The stochastic nature of collisions leads to the realization of extreme scenarios - in most cases the ion has time to transfer a small part of its energy, or a rare "successful" collision leads to the development of the collision cascade, large excitation and subsequent fragmentation of the cluster. Intermediate cases are unlikely. Consequently, the large-scale spectra of ion sputtering products will mostly be determined by "successful" collisions.

To apply the model to nanostructures, some nanoparticle characteristics need to be considered. In the case of a loose packaging of nanostructures or isolated nanostructures, the size of the excitation area can be considered nearly equal to the nanoparticle size. Using this supposition allows successful calculation of the relative yield of the clusters during fullerenes sputtering, without changing the rest of the model [1].

\section{The double sputtering mechanism of fullerenes}

In the case of closely packed nanoparticles [4] (a tablet of pressed $\mathrm{C}_{60}$ fullerene powder), the experimental data allow one to suggest the existence of two sputtering mechanisms. In the ion track, where the substance is warmed up to high temperatures and destruction of $\mathrm{C}_{60}$ molecules occurs, their structure does not influence the process of forming clusters. However outside the track, where the excitation is transferred from one molecule to another via collisions, the fullerene can be knocked out of the target as a unitary whole, and only then, it can vaporize several atoms. While calculating the impact of the additional mechanism of cluster yield, the fullerene was considered as a single particle. While dispersing the energy into targets, a depositing ion creates an excitation zone of $N_{0}$ atoms. Target atoms receive a $q$ impulse which we find equal for every atom, directed accidentally or with equal probability. The $\mathrm{C}_{n}$ relative yield of clusters from the ion track was calculated by a standard technique [1].

However, collisions cascades spread outside the ion track. Suppose from the moment of the cascade exits outside the track of the bombarding ion until the moment of decreasing of the average energy involved in the ion cascade below $E_{d}$ captures $N_{0 f}$ of fullerene molecules, transferring a $q_{f}$ effective impulse to them. The value of fullerenes' impulse knocked out beyond the ion track is obviously limited both from above $q_{f}<60 q$ and below 
$q_{f}>\sqrt{E_{d} 2 m_{f}}$. In previous work [1], the expression (4) was obtained for $W_{N}-$ the possibility of emission from the excited cluster zone consisting of $N$ atoms with internal energy $E_{\text {int }}$ :

$$
\frac{d W_{N}}{d E_{\text {int }}}=\Omega \frac{\sqrt{2\left(N q^{2} /(2 m)-E_{\text {int }}\right)}}{8 \pi^{3 / 2}(\varepsilon / 3)^{3 / 2}} \times \exp \left(-\frac{3\left(N q^{2} /(2 m)-E_{\text {int }}\right)}{2 \varepsilon}\right),
$$

where $\Omega$ is the solid angle of the cluster emission, $m$ - atom mass, $q$-average impulse, received by the atoms of the excited zone during the descent of the ion (in our case, in the ion track), $\varepsilon$ - average atom energy, $\varepsilon=q^{2} / 2 m$. Suppose the energy of the $U_{f}$ link of the fullerene is proportional to the cluster surface which contacts with neighboring clusters and equals $h E_{d}$, where $h$ is a coefficient of the target looseness; $h=1$ when fullerenes are as close to each other as possible. To calculate the probability of fragmentation for a $\mathrm{C}_{60}$ cluster at the expense of internal energy, we will integrate the spectrum of knocked out clusters with internal energy from $n \delta$ to $(n+1) \delta$ :

$$
W_{N}^{n}=\int_{n \delta}^{(n+1) \delta} \frac{d w_{N}}{d E_{i n t}}, d E_{i n t}
$$

where $\delta$ is the sublimation energy of one carbon atom of fullerene, $n$ - amount of vaporized from an overheated cluster of atoms. We will calculate the probability of the excited fullerene flying out which will turn into a cluster of $60-n$ atoms after fragmentation. Fullerenes in the target are considered uncharged. The probability of the fullerene receiving charge during fragmentation was calculated according to the technique described in the work [1]. The results of the calculation are presented in the Fig. 1a.
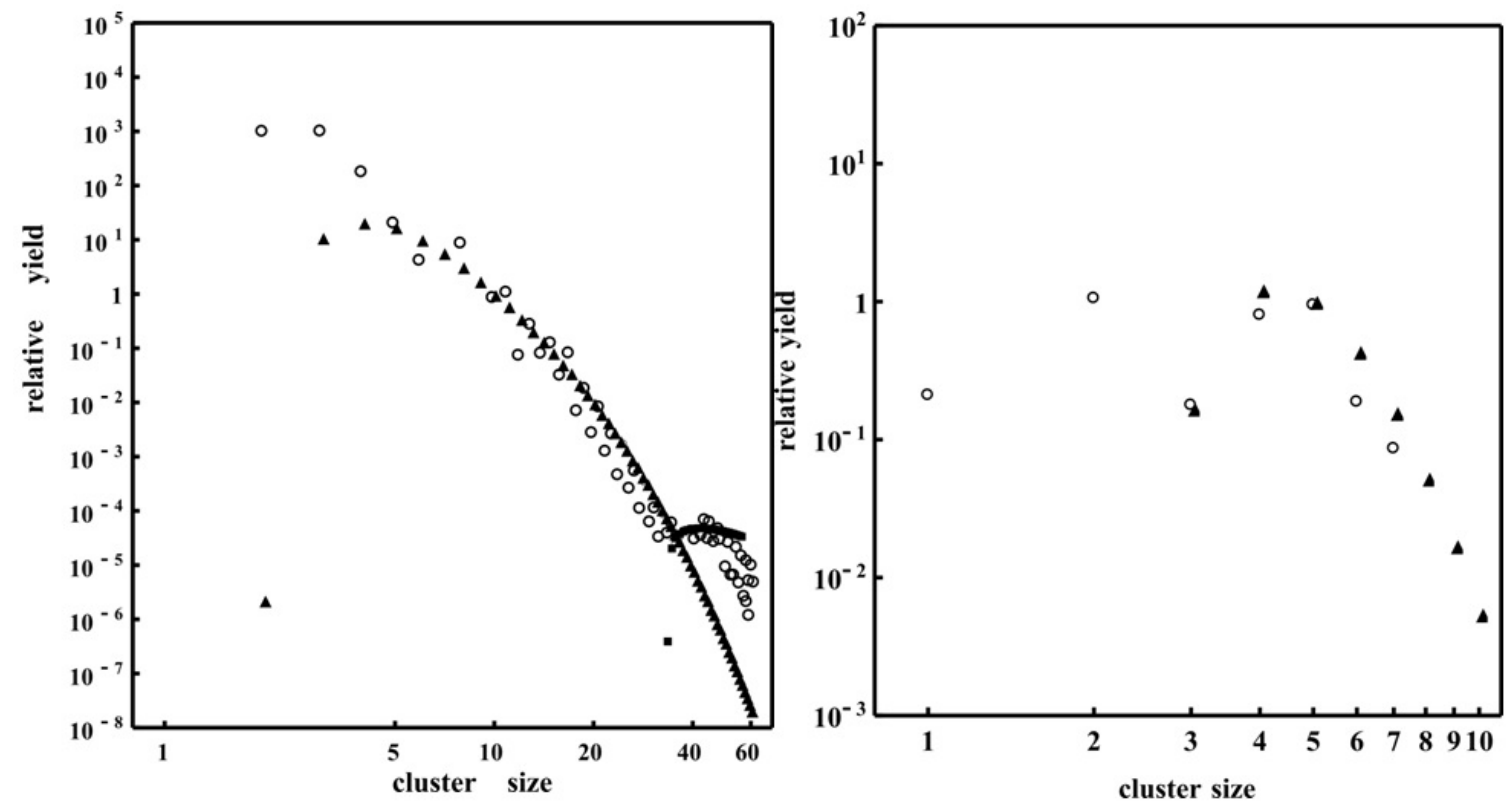

FIG. 1. Left - the relative yield of $C_{n}^{-}-$at $\mathrm{C}_{60}$ bombardment with $\mathrm{Cs}^{+} 60 \mathrm{keV}$ ions, triangle classic model, square - improved model, circle - experiment [4]. Right - the relative yield of $C_{n}^{-}$at $\mathrm{CNT}$ bombardment with $\mathrm{Cs}^{+} 5 \mathrm{keV}$ ions, triangle - model, circle - experiment [8]

\section{The sputtering mechanism of carbon nanotubes}

Carbon nanotubes have a special property which can influence the process of sputtering. This special property is ballistic thermal conductivity [5]. In a perfect CNT, a phonon moves without experiencing any sputtering. In real CNTs there are always a certain number of defects, which is why the thermal conductivity of CNTs is often quasi-ballistic or diffusive, but still remains high.

We assume during the ion bombardment of CNTs in the elastic cascade mode an increase in size of the excitation zone concerning the plain graphite. This assumption is indirectly confirmed by previous work [6], where carbon fibers, possessing a greater thermal conductivity than graphite, are shown to have a greater sputtering coefficient. 
CNTs often have inclusions in the form of metal particles. During the ion bombardment on the CNT surface metal nanoclusters can be formed. In the [7] project such CNTs are shown to have a greater sputtering coefficient than graphite. During a continuous bombardment $\mathrm{Y}$ increases. This effect is not present during the ion bombardment of graphite. This difference can be explained by the greater efficiency of transferring the impulse from the bombing ion to a metal cluster compared to that to a thin nanotube wall. Then the excitation is transferred from the cluster to the CNT. Graphite, on the other hand, is thick enough to stop the ion completely and absorb its whole impulse. The results of calculations and their comparison to the [8] experiment are shown in Fig. 1b. Calculations were conducted according to the standard model, the size of the excitation zone was considered doubled in comparison to the ordinary case.

\section{Conclusion}

It is shown that the mechanism of ion sputtering of a single nanoparticle differs very little from the mechanism for macrobodies sputtering. After the presented modernization, the model [1] has good agreement with the experimental data. It has been shown that the interaction between nanoparticles can contribute greatly in the forming of mass spectrum of clusters. Knowing the processes taking part in the ion bombardment of nanoparticles can be used for assessment of nanocoating sustainability, functional nanoaddition in composites, nanoelectronics components and also for obtaining nanoobjects functionalized with nanoclusters.

\section{References}

[1] Kapustin S.N., Matveev V.I. Emission of Charged and Stable Clusters during the Ion Sputtering of Metal. Bull. Russ. Acad. Sci., Physics, 2016, 80, P. 113-116.

[2] Kissel R., Urbassek H.M. Sputtering from spherical Au clusters by energetic atom Bombardment. Nucl. Instr. Meth. Phys. Res. B, 2001, 180, P. 293-298

[3] Zhurkin E.E. Investigation of gold nanoclaster spraying under bombarding by $\mathrm{Au}$ ions with energy $38 \mathrm{keV}$ by the classical molecular dynamics method. Surface. X-ray, Synchrotron and Neutron Studies, 2008, N3, P. 27-33.

[4] Verevkin I.V., et al. Emission and fragmentation of $\mathrm{C}_{n}$ and $\mathrm{C}_{s m} \mathrm{C}_{n}$ clusters during the foulerite bombardment with cesium ions. Pis'ma v Zhurnal Tekhnicheskoi Fiziki (Tech. Phys. Lett.), 1995, 23, P. 49-53.

[5] Eletskii A.V. Transport properties of carbon nanotubes. Phys. Usp., 2009, 52, P. 209-224.

[6] Syvorov A.L., Guseva M.I., Korshunov S.N., Zaluzhnyi A.G., Lazarev N.E. Sputtering of carbon materials with ions of hydrogen, deuterium and helium in the third-way region of energy. VANT (Problems of Atomic Science and Technology, Kharkov), 2000, 4, P. 6-9.

[7] Ahmad S., et al. Cs+ sputtered clusters from multi-walled carbon nanotubes and graphite. Nucl. Instr. Meth. Phys. Res. B, 2012, 271, P. $55-60$.

[8] Javeed S., et al. Dynamics of fragmentation and multiple vacancy generation in irradiated single-walled carbon nanotubes. Nucl. Instr. Meth. Phys. Res. B, 2013, 295, P. 22-29. 